\title{
Original
}

\section{Efficacy of p21 Expression as a Prognostic Factor for Advanced Squamous Cell Carcinomas of the Esophagus}

\author{
Satoru Goto, Masahiko MuraKami, Masahiro Waseda, \\ Tetsuo Sawatani, Atsushi Sato, Koji Otsuka, \\ Hidetoshi Makita, Kazuhiro Narita, Yoshihiro Fukoue, \\ Takashi KATO and Mitsuo KusANO
}

\begin{abstract}
The purpose of this study was to identify prognostic factors for esophageal carcinoma primary tumors and the corresponding metastatic lymph nodes by assessing tumor levels of p53, p21, p16, and cyclinD1 and comparing results with clinicopathological findings and survival rates. From December 1996 to October 2003, 69 patients with esophageal carcinoma who underwent complete thoracoscopic esophagectomies were included in this study. Immunostaining assay for $\mathrm{p} 53, \mathrm{p} 21, \mathrm{p} 16$, and cyclinD1 was performed on surgically resected main tumor and metastatic lymph node specimens. Statistical analyses were performed to determine the relationship between assayed immunoreactivity and predetermined clinicopathological findings and survival rates. Although there was no statistically significant association between p21 expression and clinicopathological findings, there was a significant difference in the 4-year survival rates between patients with p21 positive tumors and those with $\mathrm{p} 21$ negative tumors $(\mathrm{p}=0.0101)$. The survival rate of Grade 0 patients was significantly lower than other groups $(p=0.0046)$. In conclusion, the absence of p21 expression may be an important factor indicating a poor survival rate of patients with esophageal carcinoma. The absence of p21 expression in both the primary tumor and metastatic lymph nodes in advanced esophageal carcinomas indicates a worse prognosis than when expression is observed in either the primary tumor or metastatic lymph nodes. This is the first report of immunoreactivity being used to identify significant differences between patients with esophageal carcinoma primary tumors and corresponding metastatic lymph nodes that can be related to an evaluated prognosis.
\end{abstract}

Key words : esophageal carcinoma, p21, p53, p16, cyclinD1

\section{Introduction}

In general, the prognosis of patients with esophageal carcinoma is poor. For many patients with esophageal carcinoma the available treatment, which includes extended lymphandenectomy, chemotherapy, and radiotherapy, is ineffective and the survival rate is low. Various molecular factors have been investigated to grade malignancies to aid in 
prognosis and the selection of an optimal treatment regime.

Factors including p53, p21, p16, and cyclinD1 are important prognostic indicators for esophageal carcinomas. This is the first report evaluating the immunoreactivity of these factors in surgically resected primary tumor and corresponding metastatic lymph node specimens.

In this study, the immunoreactivity of p53, p21, p16 and cyclinD1 was assessed in surgically resected esophageal carcinoma main tumors and corresponding metastatic lymph node specimens, and the relationship between immunoreactivity and clinicopathological findings was examined with respect to survival rates.

\section{Patients and Methods}

From December 1996 to October 2003, 69 patients ( 57 males, 12 females; mean age 63.0 years) with esophageal carcinomas who underwent complete thoracoscopic esophagectomies in Showa University, by the same surgeon (second author) were included in this study. In all patients esophageal carcinomas were located in the left lateral decubitus and 5 ports were introduced into the right pleural cavity. A radical esophagectomy and mediastinal lymphandenectomy were carried out under thoracoscopy. An esophageal substitute was reconstructed from a gastric tube made by a laparotomy through a retrosternal route.

Tissue samples were collected retrospectively from the surgically resected specimens of all 69 patients in this study. Informed consent was obtained from all patients.

Resected specimens were fixed in $10 \%$ neutralized formalin, then cut and blocked to a thickness of $5 \mathrm{~mm}$ before embedding in paraffin. Each paraffin block was cut into $4 \mu \mathrm{m}$ thick sections with a sliding microtome. The samples were either stained with hematoxylin and eosin or subjected to immunohistochemical analysis.

The age of the 57 male and 12 female patients ranged from 41 to 87 years (mean, 63.0 years). Based on the Tumor-Node-Metastasis classification of the International Union Against Cancer ${ }^{1)}$, the tumors of the 69 patients were divided into T1, T2, T3 or T4 grade tumors. Of the 69 patients, 15 had T1 tumors, 11 had T2 tumors, 39 had T3 tumors, and three had T4 tumors. Six tumors were located in the upper third of the esophagus, 26 in the middle third, and 35 in the lower third. All patients had pathologic squamous cell carcinomas, 18 tumors were well differentiated, 36 were moderately differentiated, and 15 were poorly differentiated. Lymph node metastasis was present in 50 of the 69 patients (72.5\%) (Table 1).

\section{Immunohistochemistry}

Formalin-fixed, paraffin-embedded specimens of primary tumors and metastatic lymph node specimens were examined. After deparaffinization with xylene, and dehydration with ethanol, the specimens were treated with $0.3 \%$ hydrogen peroxide in methanol for 30 minutes to block the intrinsic peroxidase activity. Sections were then heated in $10 \mathrm{mM}$ citrate buffer at $\mathrm{pH} 6.0$ at $95^{\circ} \mathrm{C}$ for $40 \mathrm{~min}$ to retrieve the antigens. After cooling, $10 \%$ normal goat serum was placed on the sections for $30 \mathrm{~min}$ to reduce nonspecific background staining. The sections were then incubated overnight at $4^{\circ} \mathrm{C}$ in a moist chamber with the primary antibodies as follows; a mouse monoclonal anti-CyclinD1 antibody (DAKO, Clone DCS-6; dilution 1:50); a mouse monoclonal anti-p53 antibody (DAKO, Clone DO-7; dilution 1:100); a mouse monoclonal anti-p21WAF-1/CIP-1 antibody (DAKO, Clone 
Table 1. Clinicopathological findings of esophageal carcinoma

\begin{tabular}{|c|c|}
\hline & $\mathbf{n}(\%)$ \\
\hline Age & $63 \pm 7.91(41-87)$ \\
\hline Male & $57(82.6)$ \\
\hline Female & $12(17.4)$ \\
\hline \multicolumn{2}{|l|}{ Localization } \\
\hline $\mathrm{Ce}$ & $0(0)$ \\
\hline Ut & $6(8.6)$ \\
\hline Mt & $28(40.0)$ \\
\hline $\mathbf{L t}$ & $35(50.0)$ \\
\hline $\mathrm{Ae}$ & $0(0)$ \\
\hline \multicolumn{2}{|c|}{ Histological type } \\
\hline well & $22(31.4)$ \\
\hline moderately & $32(45.7)$ \\
\hline pooly & $15(21.4)$ \\
\hline \multicolumn{2}{|c|}{ Lymphatic invasion } \\
\hline $\operatorname{ly}(+)$ & $46(66.7)$ \\
\hline $1 \mathbf{y}(-)$ & $23(33.3)$ \\
\hline \multicolumn{2}{|c|}{ Vascular invasion } \\
\hline $\mathbf{v}(+)$ & $38(55.1)$ \\
\hline $\mathbf{v}(-)$ & $31(44.9)$ \\
\hline \multicolumn{2}{|c|}{ Intraepithelial spread } \\
\hline ie $(-)$ & $56(81.2)$ \\
\hline $\mathrm{ie}(+)$ & $13(18.8)$ \\
\hline \multicolumn{2}{|c|}{ Infiltrative growth pattern } \\
\hline INF $\alpha$ & $12(17.4)$ \\
\hline INF $\beta$ & $48(69.6)$ \\
\hline INF $\gamma$ & $9(13.0)$ \\
\hline \multicolumn{2}{|c|}{ Depth of invasion } \\
\hline $\mathrm{m}$ & $4(5.8)$ \\
\hline sm & $11(15.9)$ \\
\hline $\mathrm{mp}$ & $12(17.4)$ \\
\hline a1 & $39(56.5)$ \\
\hline a2 & $3(4.3)$ \\
\hline a3 & $0(0)$ \\
\hline \multicolumn{2}{|c|}{ Lymph node metastasis } \\
\hline n0 & $19(27.5)$ \\
\hline $\mathrm{n} 1$ & $10(14.5)$ \\
\hline $\mathrm{n} 2$ & $16(23.2)$ \\
\hline n3 & $8(11.6)$ \\
\hline $\mathrm{n} 4$ & $16(23.2)$ \\
\hline \multicolumn{2}{|c|}{ Clinical stage } \\
\hline 0 & $2(2.9)$ \\
\hline $\mathrm{I}$ & $8(11.6)$ \\
\hline II & $17(24.6)$ \\
\hline III & $24(34.8)$ \\
\hline IV & $18(26.1)$ \\
\hline
\end{tabular}


SX118; dilution 1:50); a mouse monoclonal anti-p16INK4a antibody (NEOMARKERS, Clone 16P07; dilution 1:500); and a mouse monoclonal anti-Ki-67 antibody (IMMUNOTECH, MIB-5; dilution 1:50). The next day, sections were treated with a secondary antibody and diaminobenzidine complex (HISTOFINE, Simple-Stein MAX-PO (MULTI)) for $30 \mathrm{~min}$ at room temperature to visualize immunoreactivity. Negative controls were prepared by omitting the antibody.

The immunohistochemical expression of each factor was evaluated by independent observers without knowledge of the corresponding clinicopathologial data. Staining was evaluated as the percentage of densely stained nuclei in 1,000 tumor cells in three random fields. When more than $10 \%$ of the tumor cells had positively stained nuclei, the specimen was scored as positive.

\section{Statistical analysis}

Statistical analysis was performed using the $\chi^{2}$ test, the Fisher's $U$ test, or the MannWhitney $U$ test for group differences. Survival rates were estimated using the Kaplan-Meier method, and the analysis was evaluated by the log-rank test. $\mathbf{P}<0.05$ was considered statistically significant.

\section{Results}

Immunohistochemical detection of p53, p21, p16, and cyclinD1, in the primary tumor and corresponding lymph node metastasis of esophageal carcinoma Primary tumor

The primary tumor samples of 27 patients were positive for cyclinD1 $(39.1 \%)$ and 42 were negative $(60.9 \%)$; 37 were positive for p53 (53.6\%) and 32 were negative $(46.4 \%)$; 25 were positive for p21 $(36.2 \%)$ and 44 were negative $(63.8 \%)$; and 19 were positive for p16 $(27.5 \%)$ and 56 were negative $(72.5 \%)$.

Primary tumor and corresponding lymph node metastasis

Pathologically, 50 out of the 69 patients with esophageal carcinoma had lymph node metastasis. We classified these patients according to the primary tumor and lymph node immunoreactivity for p53, p21, p16 and cyclinD1 as follows: Grade 0, patients negative for primary tumor and lymph nodes, Grade I, patients positive for primary tumor and negative for lymph nodes, Grade II, patients negative for primary tumor and positive for lymph nodes, Grade III, patients positive for both primary tumors and lymph nodes.

For cyclinD1, 17 patients were Grade 0, 12 were Grade I, four were Grade II, and 17 were Grade III. For p53, 22 patients were Grade 0, 10 were Grade I, two were Grade II, and 15 were Grade III. For p21, 12 patients were Grade 0, nine were Grade I, four were Grade II, and 25 were Grade III. For p16, 33 patients were Grade 0, two were Grade I, two were Grade II, and 13 were Grade III.

\section{Relationship between Immunoreactivity and Clinicopathological Findings Primary tumor}

No statistically significant relationship was observed between p53 p21, p16, or CyclinD1, expression in primary tumors and the clinicopathological findings (Table 2).

Primary tumor and corresponding lymph node metastasis

No significant differences were found between the four groups of graded patients (Grade 
Table 2. Relationship between p53, p21, p16, and cyclinD1 expression and clinicopathological findings. No statistically significant association was observed in the relationships between p53, p21, p16, and cyclinD1 expression and clinicopathological findings

\begin{tabular}{|c|c|c|c|c|c|c|}
\hline \multirow[t]{2}{*}{ I } & \multicolumn{6}{|c|}{ Relationship between the expression of $\mathrm{p} 53, \mathrm{p} 21$ and Clinicopathological Findings } \\
\hline & $\begin{array}{c}\mathrm{p} 53(+) \\
\mathrm{N}=37\end{array}$ & $\begin{array}{c}\mathrm{p} 53(-) \\
\mathrm{N}=32\end{array}$ & $p$ value & $\begin{array}{c}\mathrm{p} 21(+) \\
\mathrm{N}=25\end{array}$ & $\begin{array}{c}\mathrm{p} 21(-) \\
\mathrm{N}=44\end{array}$ & $p$ value \\
\hline Age & & & ns $(a)$ & & & ns $(\mathbf{a})$ \\
\hline$<60$ & 15 & 13 & & 10 & 18 & \\
\hline$\geqq 60,<70$ & 14 & 12 & & 11 & 15 & \\
\hline$\geqq 70$ & 8 & 7 & & 4 & 11 & \\
\hline Sex & & & ns $(b)$ & & & ns $(b)$ \\
\hline Male & 30 & 27 & & 19 & 38 & \\
\hline Female & 7 & 5 & & 6 & 6 & \\
\hline \multicolumn{7}{|l|}{ Localization } \\
\hline $\mathrm{Ce}$ & & & ns $(b)$ & & & ns $(b)$ \\
\hline Ut & 4 & 2 & & 1 & 5 & \\
\hline Mt & 15 & 13 & & 12 & 16 & \\
\hline $\mathbf{L t}$ & 18 & 17 & & 12 & 23 & \\
\hline \multicolumn{7}{|l|}{$\mathrm{Ae}$} \\
\hline Histological type & & & ns (b) & & & ns (b) \\
\hline well & 7 & 11 & & 5 & 13 & \\
\hline moderately & 25 & 11 & & 15 & 21 & \\
\hline pooly & 5 & 10 & & 5 & 10 & \\
\hline Lymphatic invasion & & & ns $(a)$ & & & ns $(a)$ \\
\hline $\operatorname{ly}(+)$ & 27 & 19 & & 16 & 30 & \\
\hline $1 \mathbf{y}(-)$ & 10 & 13 & & 9 & 14 & \\
\hline \multirow{3}{*}{$\begin{array}{l}\text { Vascular invasion } \\
\mathbf{v}(+) \\
\mathbf{v}(-)\end{array}$} & & & ns $(a)$ & & & ns $(b)$ \\
\hline & 22 & 16 & & 13 & 25 & \\
\hline & 15 & 16 & & 12 & 19 & \\
\hline \multirow{4}{*}{$\begin{array}{l}\text { Intraepithelial spread } \\
\mathrm{ie}(+) \\
\mathrm{ie}(-) \\
\text { Infiltrative growth pattern }\end{array}$} & & & ns $(a)$ & & & ns $(a)$ \\
\hline & 8 & 5 & & 4 & 9 & \\
\hline & 29 & 27 & & 21 & 35 & \\
\hline & & & ns $(b)$ & & & ns $(b)$ \\
\hline INF $\alpha$ & 9 & 9 & & 9 & 9 & \\
\hline INF $\beta$ & 24 & 21 & & 13 & 32 & \\
\hline INF $\gamma$ & 4 & 2 & & 3 & 3 & \\
\hline Depth of invasion & & & ns $(c)$ & & & ns $(c)$ \\
\hline Tis & 0 & 2 & & 1 & 1 & \\
\hline $\mathrm{T} 1$ & 8 & 5 & & 6 & 7 & \\
\hline $\mathrm{T} 2$ & 5 & 7 & & 7 & 5 & \\
\hline T3 & 22 & 17 & & 10 & 29 & \\
\hline $\mathrm{T} 4$ & 2 & 1 & & 1 & 2 & \\
\hline Lymph node metastasis & & & ns $(\mathrm{c})$ & & & ns (c) \\
\hline no & 9 & 10 & & 7 & 12 & \\
\hline $\mathrm{n} 1$ & 4 & 6 & & 5 & 5 & \\
\hline $\mathrm{n} 2$ & 11 & 5 & & 5 & 11 & \\
\hline n3 & 5 & 3 & & 3 & 5 & \\
\hline $\mathrm{n} 4$ & 8 & 8 & & 5 & 11 & \\
\hline Clinical stage & & & ns $(c)$ & & & ns $(c)$ \\
\hline 0 & 1 & 1 & & 2 & 0 & \\
\hline $\mathrm{I}$ & 4 & 4 & & 3 & 5 & \\
\hline II & 6 & 11 & & 7 & 10 & \\
\hline III & 16 & 8 & & 6 & 18 & \\
\hline \multirow[t]{2}{*}{ IV } & 10 & 8 & & 7 & 11 & \\
\hline & $\begin{array}{l}\text { a; } \times 2 \text { te } \\
\text { b; Fisher }\end{array}$ & $\begin{array}{r}\mathrm{c} ; \\
\mathrm{ns}:\end{array}$ & $\begin{array}{l}\text { n-Whitn } \\
\text { significar }\end{array}$ & test & & \\
\hline
\end{tabular}


Satoru Goto, et al

Continue from Table 2

\begin{tabular}{|c|c|c|c|c|c|c|}
\hline \multirow[t]{3}{*}{ II } & \multicolumn{6}{|c|}{ Relationship between the expression of cyclinD1, p16 and clinicopathological findings } \\
\hline & \multirow{2}{*}{$\begin{array}{c}\mathrm{p} 16(+) \\
\mathrm{N}=19\end{array}$} & \multirow{2}{*}{$\begin{array}{c}\mathrm{p} 16(-) \\
\mathrm{N}=50\end{array}$} & \multirow{2}{*}{$p$ value } & \multicolumn{2}{|c|}{ CyclinD1 ( + CyclinD1 (-) } & \multirow{2}{*}{$\mathrm{p}$ value } \\
\hline & & & & $\mathrm{N}=42$ & $\mathrm{~N}=27$ & \\
\hline Age & & & ns $(a)$ & & & ns (a) \\
\hline$<60$ & 10 & 18 & & 17 & 11 & \\
\hline$\geqq 60,<70$ & 6 & 20 & & 15 & 11 & \\
\hline$\geqq 70$ & 3 & 12 & & 10 & 5 & \\
\hline Sex & & & ns $(b)$ & & & ns $(b)$ \\
\hline Male & 17 & 40 & & 36 & 21 & \\
\hline Female & 2 & 10 & & 6 & 6 & \\
\hline Localization & & & & & & \\
\hline $\mathrm{Ce}$ & & & ns $(b)$ & & & ns (b) \\
\hline Ut & 2 & 4 & & 4 & 2 & \\
\hline Mt & 8 & 20 & & 16 & 12 & \\
\hline $\mathbf{L t}$ & 9 & 26 & & 22 & 13 & \\
\hline $\mathrm{Ae}$ & & & & & & \\
\hline Histological type & & & ns $(b)$ & & & ns $(b)$ \\
\hline well & 3 & 15 & & 9 & 9 & \\
\hline moderately & 12 & 24 & & 24 & 12 & \\
\hline pooly & 4 & 11 & & 9 & 6 & \\
\hline Lymphatic invasion & & & ns $(\mathbf{a})$ & & & ns $(a)$ \\
\hline $\operatorname{ly}(+)$ & 11 & 35 & & 29 & 17 & \\
\hline $\operatorname{ly}(-)$ & 8 & 15 & & 13 & 10 & \\
\hline Vascular invasion & & & ns $(\mathbf{a})$ & & & ns $(\mathbf{a})$ \\
\hline $\mathbf{v}(+)$ & 12 & 26 & & 25 & 13 & \\
\hline $\mathbf{v}(-)$ & 7 & 24 & & 17 & 14 & \\
\hline Intraepithelial spread & & & ns $(\mathbf{a})$ & & & ns $(a)$ \\
\hline $\mathrm{ie}(+)$ & 3 & 10 & & 7 & 6 & \\
\hline $\mathrm{ie}(-)$ & 16 & 40 & & 35 & 21 & \\
\hline Infiltrative growth pattern & & & ns $(b)$ & & & ns $(b)$ \\
\hline INF $\alpha$ & 7 & 11 & & 9 & 8 & \\
\hline INF $\beta$ & 10 & 35 & & 28 & 18 & \\
\hline INF $\gamma$ & 2 & 4 & & 5 & 1 & \\
\hline Depth of invasion & & & ns (c) & & & ns $(\mathbf{c})$ \\
\hline Tis & 0 & 2 & & 2 & 0 & \\
\hline $\mathrm{T} 1$ & 2 & 11 & & 8 & 5 & \\
\hline $\mathrm{T} 2$ & 4 & 8 & & 5 & 7 & \\
\hline $\mathrm{T} 3$ & 13 & 26 & & 25 & 14 & \\
\hline $\mathrm{T} 4$ & 0 & 3 & & 2 & 1 & \\
\hline Lymph node metastasis & & & ns $(c)$ & & & ns (c) \\
\hline 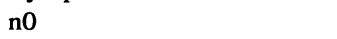 & 3 & 16 & & 13 & 6 & \\
\hline $\mathrm{n} 1$ & 4 & 6 & & 7 & 3 & \\
\hline $\mathrm{n} 2$ & 4 & 12 & & 11 & 5 & \\
\hline n3 & 4 & 4 & & 3 & 5 & \\
\hline $\mathrm{n} 4$ & 4 & 12 & & 8 & 8 & \\
\hline Clinical stage & & & ns $(c)$ & & & ns (c) \\
\hline 0 & 1 & 1 & & 1 & 1 & \\
\hline I & 1 & 7 & & 5 & 3 & \\
\hline II & 4 & 13 & & 10 & 7 & \\
\hline III & 8 & 16 & & 17 & 7 & \\
\hline \multirow[t]{2}{*}{ IV } & 5 & 13 & & 9 & 9 & \\
\hline & $\begin{array}{l}\mathrm{a} ; \times 2 \text { test } \\
\mathrm{b} ; \text { Fisher's test }\end{array}$ & $\begin{aligned} c & ; \\
\text { ns : } & \mathbf{n}\end{aligned}$ & $\begin{array}{l}\text { Whitney } \\
\text { nificant }\end{array}$ & test & & \\
\hline
\end{tabular}




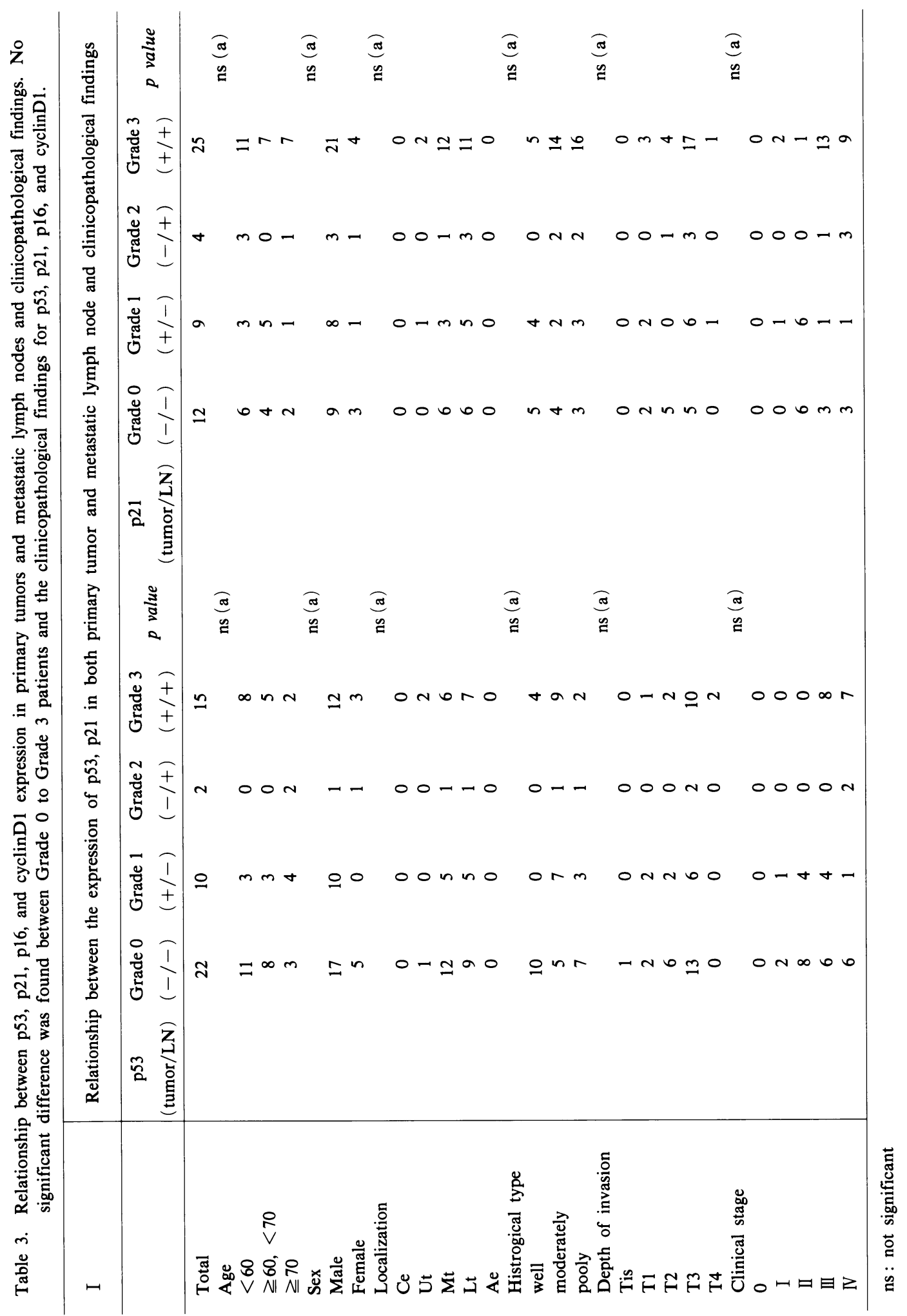




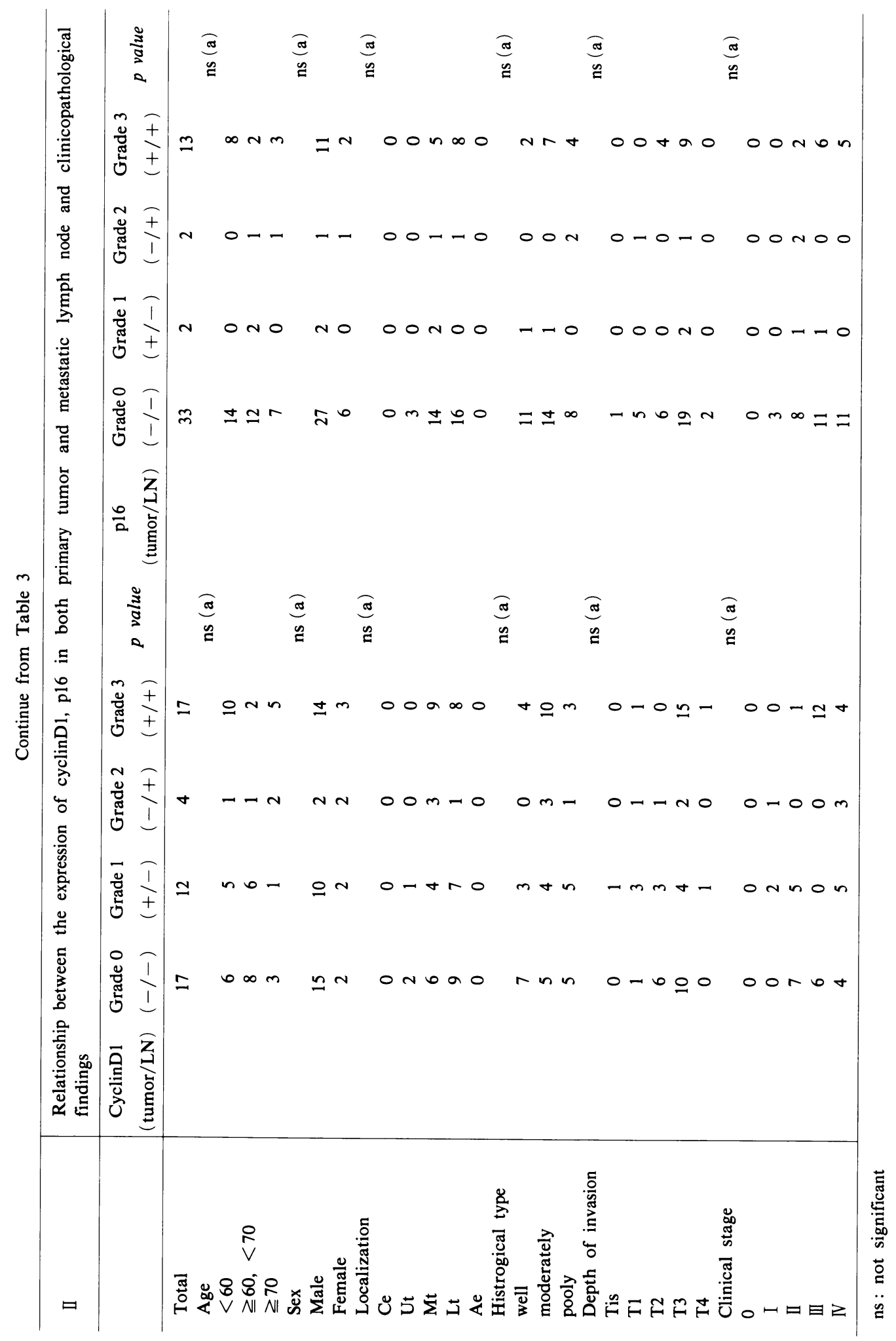




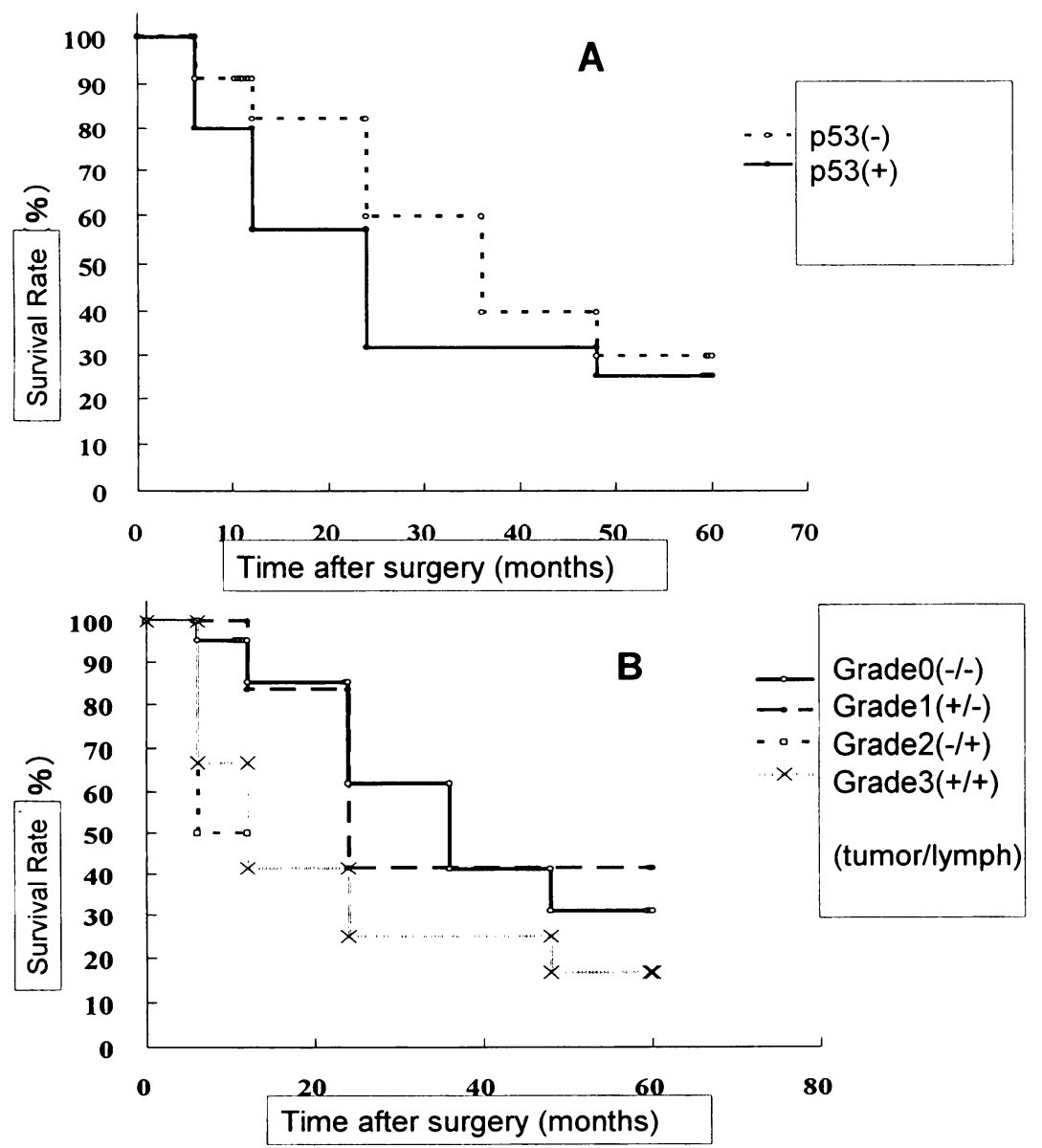

Fig. 1.

A: Kaplan-Meier survival curves of overall survival of patients compared with expression of p53 in primary tumors. There were no significant differences in the survival rate between p53 positive and negative patients $(p=0.1299)$

B: Kaplan-Meier survival curves of overall survival for the patients compared with expression of p53 in primary tumors and metastatic lymph nodes. There was no significant difference in survival rate between Grade 0 to Grade 3 patients $(p=0.1251)$

0 to Grade 3) and the clinicopathological findings for p53, p21, p16 or Cyclin D1 (Table 3 ).

Correlation with the postoperative cumulative survival rate

The 1-, 2-, 3-, and 4-year survival rates of all patients were $75.0 \%, 52.3 \%, 44.8 \%$, and $39.8 \%$, respectively.

Relationship between immunoreactivity and postoperative survival rate

Primary tumor

The 1-, 2-, 3-, and 4-year survival rates of patients with p53 positive primary tumors were $67.3 \%, 41.1 \%, 37.4 \%$, and $33.7 \%$, respectively, and the corresponding survival rates of 

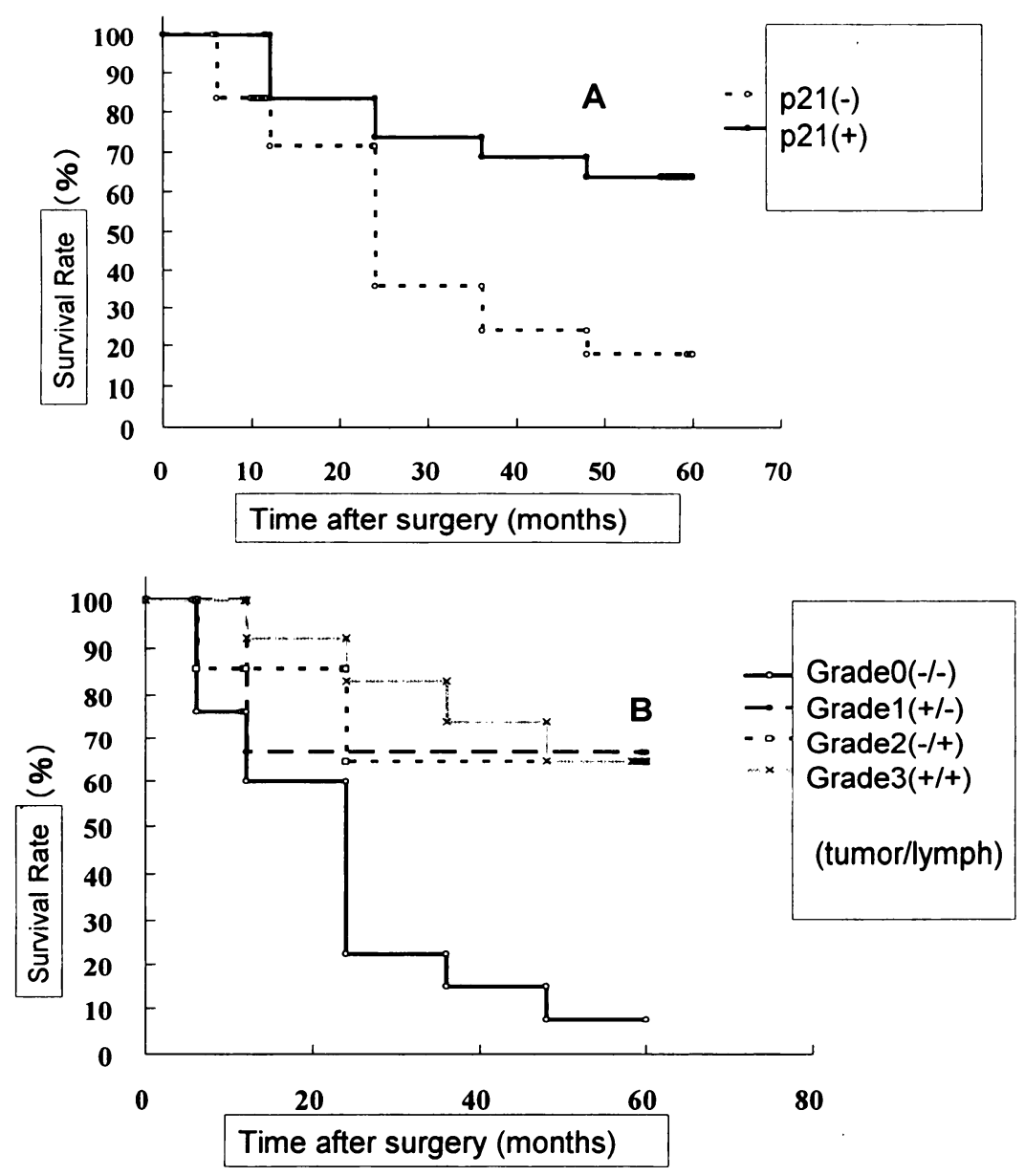

Fig. 2.

A: Kaplan-Meier survival curves of patients compared with expression of P21 in primary tumors. The 4-year survival rate of patients with the p21 negative tumors was significantly lower than patients with p21 positive tumors $(p=0.0101)$

B: Kaplan-Meier survival curves for the patients compared with expression of p21 in primary tumors and metastatic lymph nodes. The 4-year survival rate of Grade 0 patients (p21 negative both primary tumor and metastatic lymph node) was significantly lower than the other groups $(p=0.0045)$

patients with p53 negative primary tumors were $85.9 \%, 68.8 \%, 55.0 \%$, and $48.1 \%$. Therefore, there is no significant difference in the survival rate between patients with p53 positive or negative primary tumors (Fig 1-A).

The 1-, 2-, 3-, and 4-year survival rates of patients with p21 positive tumors were $82.6 \%$, $72.3 \%, 67.1 \%$, and $62.0 \%$, respectively, and the corresponding survival rates of patients with p21 negative primary tumors were $72.2 \%, 38.2 \%, 27.3 \%$, and $21.9 \%$. Therefore, there is a significant difference in the 4-year survival rate between patients with p21 -positive and -negative tumors $(p=0.0101)$ (Fig 2-A).

The 1-, 2-, 3-, and 4-year survival rates of patients with cyclinD1 positive tumors were 


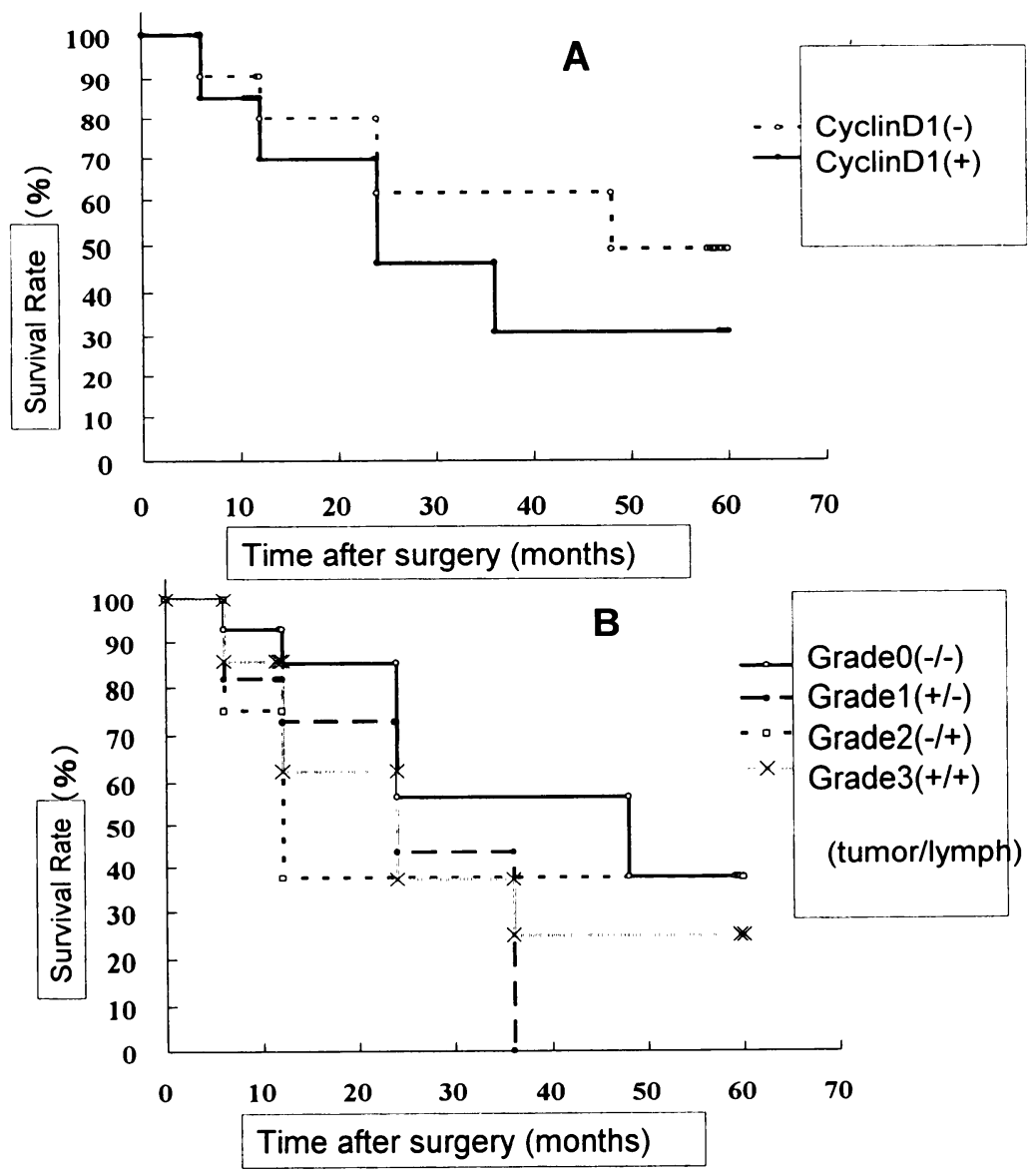

Fig. 3.

A: Kaplan-Meier survival curves of overall survival of patients compared with expression of cyclinD1 in primary tumors. There was no significant difference in the survival rate between patients with cyclinD1 positive or negative tumors $(p=0.5414)$

B: Kaplan-Meier survival curves of overall survival of patients compared with expression of cyclinD1 in primary tumors and metastatic lymph nodes. There was no significant difference in survival rate between the groups (Grade 0 to Grade 3$)(p=0.6518$ )

$78.0 \%, 49.3 \%, 34.5 \%$, and $34.5 \%$, respectively, and the corresponding survival rates of patients with cyclinD1 negative primary tumors were $73.3 \%, 57.6 \%, 57.6 \%$, and $47.1 \%$. Therefore, there is no significant difference in the survival rate between patients with cyclinD1 positive and negative tumors (Fig 3-A).

The 1-, 2-, 3-, and 4-year survival rates of patients with p16 positive tumors were $87.1 \%$, $55.4 \%, 47.5 \%$, and $31.7 \%$, respectively, and the corresponding survival rates of patients with p16 negative primary tumors were $72.5 \%, 52.7 \%, 45.2 \%$, and $45.2 \%$. Therefore, there is no significant difference in the survival rate between patients with p16 -positive and -negative tumors (Fig 4-A).

Primary tumor and corresponding lymph node metastasis

For p53, the 4-year survival rates of Grade 0, 1, 2, and 3 patients were $35.5 \%, 41.7 \%$, 

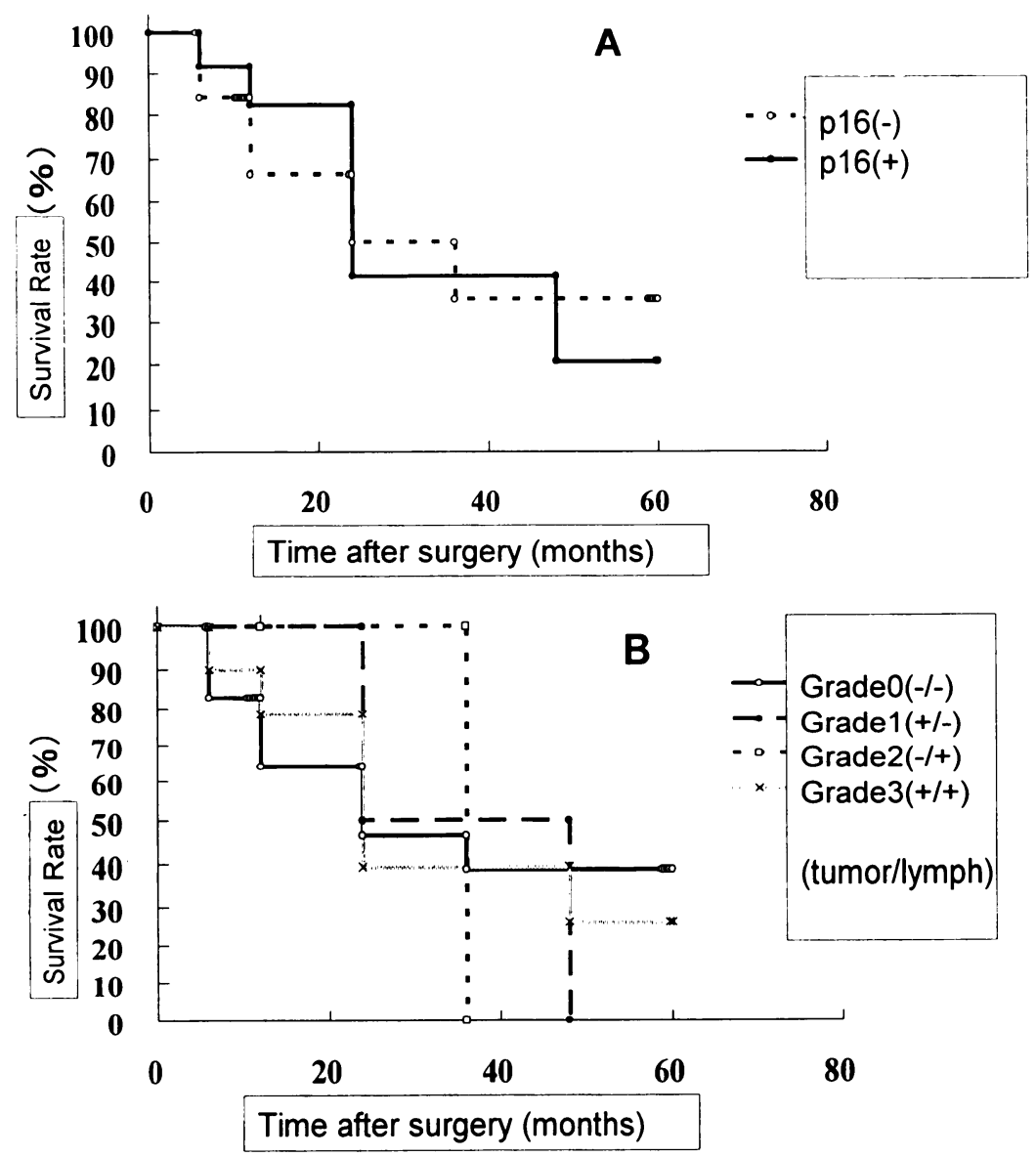

Fig. 4.

A : Kaplan-Meier survival curves of overall survival of patients compared with expression of p16 in primary tumors. There was no significant difference in survival rate between patients with p16 positive or negative tumors $(p=0.9034)$

B: Kaplan-Meier survival curves of overall survival of patients compared with expression of p16 in primary tumors and metastatic lymph nodes. There was no significant difference in survival rate between the groups (Grade 0 to Grade 3) $(p=0.9939)$

$0 \%$, and $16.7 \%$, respectively. Therefore, there is no significant difference in survival rate between Grades with respect to p53 expression (Fig 1-B).

For p21, the 4-year survival rates of Grade 0, 1, 2, and 2 patients were $7.5 \%, 66.7 \%$, $64.3 \%$, and $64.6 \%$, respectively. The survival rate of Grade 0 patients is significantly lower than the other Grades with respect to p21 expression $(p=0.0046)$ (Fig 2-B).

For cyclinD1, the 4-year survival rates of Grade $0,1,2$, and 3 patients were $37.8 \%, 0 \%$, $37.5 \%$, and $24.9 \%$, respectively. Therefore, there is no significant difference in survival rate between Grades with respect to cyclinD1 expression (Fig 3-B).

For p16, the 4-year survival rates of Grade 0, 1, 2, and 3 patients were $38.8 \%, 0 \%, 0 \%$, and $26.3 \%$. There were no significant differences in the survival rates for groups (Fig 4-B). 


\section{Discussion}

The prognosis for patients with esophageal carcinoma is poor and the postoperative survival rate is lower than for other digestive system cancers. Although various treatment options are available, such as curative surgery, chemotherapy, radiotherapy, or a combination of these therapy options, few centers have reported more than a $20 \% 5$-year survival rate $^{2)}$.

The characteristics of esophageal cancer are generally determined according to histopathological findings. A disadvantage of this approach is that esophageal cancers that are histologically similar to squamous cell carcinoma have been reported to have different biological characteristics that include differential sensitivity to neoadjuvant therapy. The survival period and quality of life after surgery are important factors in the determination of treatment options. If tumor characteristics can be determined from surgical specimens early on in the treatment regime more effective treatment options can be selected on an individual basis. Therefore, we sought to determine biological malignancy using immunohistochemical techniques and compared findings to various clinicopathological factors and prognosis.

Although p53, p21, p16, and cyclinD1 are known prognostic factors for esophageal squamous cell carcinoma, the immunoreactivity of these factors in primary tumors and corresponding metastatic lymph nodes has not been evaluated.

p53 is related to $G 1$ phase cell cycle arrest and apoptosis ${ }^{3-5)}$, and plays an important roll in cell proliferation and differentiation. Mutations impairing p53 function are a frequent event in cancer. Since the cellular half-life of the protein molecule is very short and cellular levels are low, the normal mucosa is not stained using monoclonal antibodies against the p53 protein. By comparison, mutant p53 proteins can have longer half-lives and be readily detected by immunostaining ${ }^{6)}$. The reported percentage of patients with p53 positive esophageal cancer based on immunohistochemical analysis ranges from $45 \%$ to $74 \%{ }^{7-9)}$. In the present study, $53.6 \%$ of esophageal cancers were p53 positive and this result falls within the expected range. We found no significant correlation between immunostaining of p53 and clinicopathological findings. Also, we found no significant difference in the survival rate between $\mathrm{p} 53$ positive and negative patients. Some studies report no clear relationship between abnormal p53 expression and various pathological factors $^{10,11)}$, while others noted a positive relationship ${ }^{12,13)}$. Inada et al suggest that this difference is due to differences in the antibody used, staining methods, or criteria used to classify tumors ${ }^{10)}$.

The cyclin dependent kinase inhibitor known as p21 is a downstream effector of p53 dependent cell cycle regulation. In general, positive p21 expression relates to a favorable prognosis and effective chemotherapy, while negative expression relates to a poor prognosis and ineffective chemotherapy ${ }^{14,15)}$. Our results show no significant correlation between immunostaining of p21 and clinicopathological findings. Conversely, when 4-year survival rates were compared with p21 immunoreactivity in primary tumors survival rates of patients with p21 negative tumors were significantly lower than patients with p21 positive tumors $(p=0.0101)$. Furthermore, the 4-year survival rate of Grade 0 patients ( $\mathrm{p} 21$ negative both primary tumor and metastatic lymph node) was significantly lower than the other grades of patients $(p=0.0045)$. Although we found no significant difference between primary tumor 
and corresponding metastatic lymph node immunostaining of p21 and clinicopathological findings, the independent study investigating survival rate of patients with p21 negative primary tumors and metastatic lymph nodes found that the 4-year survival rate was only $7.5 \%$ and was significantly lower than other grades of patients. While p53-dependent p21 protein expression is useful for predicting prognosis ${ }^{14,15)}$, recent studies highlight P53independent p21 protein expression as playing a central role in cell growth regulation and apoptosis and report that the p53-independent p21 protein is not mutant p53 dependent, but wild-type p53 dependent ${ }^{16,17)}$. Our results showing that p21 immunoreactivity had a significant impact on survival rate independent of p53 immunoreactivity are consistent with these latter reports.

Cyclin D1 is a cell-regulatory protein expressed at high levels during the G1 phase of the cell cycle ${ }^{18)}$. The reported percentage of patients with cyclinD1 positive esophageal cancer based on immunohistochemical analysis ranges from $22 \%$ to $42 \%{ }^{18-20}$. The percentage of patients in our study with cyclinD1 positive esophageal cancers was $39.1 \%$ and this result stands within the expected range. cyclinD1 amplification and overexpression is reported in a variety of tumors. cyclinD1 overexpression induces increased tumor cell proliferation and esophageal carcinomas overexpressing cyclinD1 aggressively invade lymphatic vessels and metastasize to lymph nodes, but do not invade blood vessels or other organs ${ }^{21)}$. Details of the underlying mechanisms involving cyclinD1 remain unclear ${ }^{21)}$. In the present study we found no significant correlation between cyclinD1 immunostaining and the clinicopathological findings. There was also no significant difference in survival rate between cyclinD1 positive and negative patients.

Factor p16 is involved in G1 cell cycle arrest and is upregulated in cellular stress such as DNA damage. It is thought to act as an important tumor suppressor ${ }^{22)}$. Mutation of the p16 gene may occur in cancer cells resulting in a reduction or loss of p16 expression ${ }^{23)}$. In our study, no significant correlation was found between immunostaining of p16 and clinicopathological findings. There was also no significant difference in survival rate between p16 positive and negative patients.

\section{References}

1) Sobin LH and Fleming ID: TNM Classification of Malignant Tumors, fifth edition. Cancer 80 : $1803-1804$ (1997)

2) Walsh TN, Noonan N, Hollywood D, Kelly A, Keeling N and Hennessy TP : A comparison of multimodal therapy and surgery for esophageal adenocarcinoma. N Engl J Med 335 : 462-467 (1996)

3) Brugarolas J, Chandrasekaran C, Gordon JI, Beach D, Jacks T and Hannon GJ : Radiation-induced cell cycle arrest compromised by p21 deficiency. Nature $377: 552-557$ (1995)

4) Waldman T, Lengauer C, Kinzler KW and Vogelstein B: Uncoupling of S phase and mitosis induced by anticancer agents in cells lacking p21. Nature $381: 713-716$ (1996)

5) Moreira LF, Naomoto Y, Hamada M, Kamikawa Y and Orita K : Assessment of apoptosis in oesophageal carcinoma preoperatively treated by chemotherapy and radiotherapy. Anticancer Res 15:639-644 (1995)

6) Finlay CA, Hinds PW, Tan TH, Eliyahu D, Oren $\mathbf{M}$ and Levine AJ : Activating mutations for transformation by p53 produce a gene product that forms an hsc70-p53 complex with an altered half-life. Mol Cell Biol 8 : 531-539 (1988)

7) Wagata T, Shibagaki I, Imamura M, Shimada Y, Toguchida J, Yandell DW, Ikenaga M, Tobe T and Ishizaki $\mathrm{K}$ : Loss of $17 \mathrm{p}$, mutation of the p53 gene, and overexpression of p53 protein in esophageal squamous cell carcinomas. Cancer Res 53 : 846-850 (1993)

8) Murakami S, Uchida Y, Takeno S, Noguchi T, Matsumoto K and Shimoda H : Expression of PCNA and p53 in esophageal dysplasia and esophageal carcinoma. Surg Today 27 : 593-599 (1997) 
9) Parenti AR, Rugge M, Frizzera E, Ruol A, Noventa F, Ancona E and Ninfo V: p53 overexpression in the multistep process of esophageal carcinogenesis. Am J Surg Pathol 19 : 1418-1422 (1995)

10) Inada $S$, Koto $T$, Futami $K$, Arima $S$ and Iwashita $A$ : Evaluation of malignancy and the prognosis of esophageal cancer based on an immunohistochemical study (p53, E-cadherin, epidermal growth factor receptor). Surg Today $29:$ 493-503 (1999)

11) Sarbia M, Porschen R, Borchard F, Horstmann O, Willers R and Gabbert HE : p53 protein expression and prognosis in squamous cell carcinoma of the esophagus. Cancer $74: 2218-2223$ (1994)

12) Goukon Y, Sasano H, Nishihira T, Nagura H and Mori S : p53 overexpression in human esophageal carcinoma : a correlation with tumor DNA ploidy and two parameter flow cytometric study. Anticancer Res $14: 1305-1312$ (1994)

13) Shimaya $K$, Shiozaki $H$, Inoue $M$, Tahara $H$, Monden $T$, Shimano $T$ and Mori $T$ : Significance of p53 expression as a prognostic factor in oesophageal squamous cell carcinoma. Virchows Arch A Pathol Anat Histopathol 422 : 271-276 (1993)

14) Natsugoe S, Nakashima S, Matsumoto M, Xiangming C, Okumura H, Kijima F, Ishigami S, Takebayashi Y, Baba M, Takao S and Aikou T: Expression of p21WAF1/Cip1 in the p53-dependent pathway is related to prognosis in patients with advanced esophageal carcinoma. Clin Cancer Res 5 : 2445-2449 (1999)

15) Nakashima S, Natsugoe S, Matsumoto M, Kijima F, Takebayashi Y, Okumura H, Shimada M, Nakano S, Kusano C, Baba M, Takao S and Aikou T: Expression of p53 and p21 is useful for the prediction of preoperative chemotherapeutic effects in esophageal carcinoma. Anticancer Res 20 : 1933-1937 (2000)

16) Yasui W, Akama Y, Kuniyasu H, Yokozaki H, Semba S, Shimamoto F and Tahara E: Expression of cyclin-dependent kinase inhibitor p21WAF1/CIP1 in non-neoplastic mucosa and neoplasia of the stomach: relationship with p53 status and proliferative activity. J Pathol 180 : 122-128 (1996)

17) Giannikaki E, Kouvidou C, Tzardi M, Stefanaki K, Koutsoubi K, Gregoriou M, Zois E, Kakolyris S, Mavroudi C, Delides G, Georgoulias V and Kanavaros P: p53 protein expression in breast carcinomas. Comparative study with the wild type p53 induced proteins mdm2 and p $21 /$ waf1. Anticancer Res. May-Jun, $17: 2123-2127$ (1997)

18) Toyoda H, Nakamura T, Shinoda M, Suzuki T, Hatooka S, Kobayashi S, Ohashi K, Seto M, Shiku H and Nakamura S : Cyclin D1 expression is useful as a prognostic indicator for advanced esophageal carcinomas, but not for superficial tumors. Dig Dis Sci 45 : 864-869 (2000)

19) Naitoh H, Shibata J, Kawaguchi A, Kodama $M$ and Hattori T: Overexpression and localization of cyclin D1 mRNA and antigen in esophageal cancer. Am J Pathol 146 : 1161-1169 (1995)

20) Gramlich TL, Fritsch CR, Maurer D, Eberle $M$ and Gansler TS : Differential polymerase chain reaction assay of cyclin D1 gene amplification in esophageal carcinoma. Diagn Mol Pathol 3 : 255-259 (1994)

21) Nagasawa S, Onda M, Sasajima K, Makino H, Yamashita K, Takubo K and Miyashita M: Cyclin D1 overexpression as a prognostic factor in patients with esophageal carcinoma. J Surg Oncol 78 : 208-214 (2001)

22) Sharpless NE and DePinho RA: The INK4A/ARF locus and its two gene products. Curr Opin Genet Dev 9 : 22-30 (1999)

23) Sturm I, Petrowsky H, Volz R, Lorenz M, Radetzki S, Hillebrand T, Wolff G, Hauptmann S, Dorken B and Daniel PT: Analysis of p53/BAX/p16 (ink4a/CDKN2) in esophageal squamous cell carcinoma: high BAX and p16 (ink4a/CDKN2) identifies patients with good prognosis. J Clin Oncol 19 : 2272-2281 (2001)

[Received February 9, 2004 : Accepted February 19, 2004] 